\title{
Note on Pareto Optimality and Duality for Certain Nonlinear Systems
}

\section{Werner Oettli}

Nr. $85(1988)$

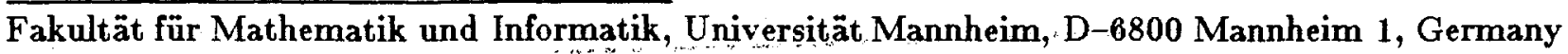




\title{
Note on Pareto Optimality and Duality \\ for Certain Nonlinear Systems
}

\author{
Werner Oettli ${ }^{1}$
}

\begin{abstract}
Abotract. We characterize the inconsistency of certain nonlinear systems under mild convexity requirements and without need for a regularity assumption. The result is used to establish a duality result for Pareto optimal points.
\end{abstract}

1. The present note is a continuation of [5]. There for $Y$ a real topological vector space, $P \subset Y$ a nonvoid convex cone, and $S \subset Y \times \mathbb{R}$ a nonvoid set, the inconsistency of the system

$$
(y, t) \in S, \quad y \in-P, \quad t<0
$$

had been characterized by the existence of $y^{*} \in P^{*} \quad\left(P^{*}\right.$ the polar cone of $\left.P\right)$ such that

$$
0 \leq t \text { for all }(y, t) \in S \text { satisfying }\left\langle y^{*}, y\right\rangle \leq 0 .
$$

In order to make the necessity part of this characterization valid one needs first a convexity assumption, namely that the set $D:=\{y \in Y \mid(y, t) \in S, t<0\}$ is convex, and second one needs a so called regularity assumption, which may take various forms. The simplest regularity assumption, but also the least practical for many applications, requires the set $D$ to be open in $Y$. Another regularity assumption, which in essence goes back to [3], requires that $D$ is open in $S_{Y}$ ( $S_{Y}$ the projection of $S$ onto $Y$ ) and $S_{Y}$ is convex with $0_{Y} \in$ int $S_{Y}$. This assumption is more practical, but still has its drawbacks. Here, similarly to [1], we want to describe a simple approach which does not need any regularity assumption at all, yet gives a necessary and sufficient condition for the inconsistency of the above system. $\mathbb{R}$ is replaced by a more general vector space $Z$, permitting the consideration of Pareto optima. We conclude with a duality result in scalar and vectorial form respectively.

2. From now on we shall make the following assumptions :

$Y, Z$ are real topological vector spaces, with $Y$ being locally convex;

$P \subset Y$ and $Q \subset Z$ are nonvoid convex cones, with $P$ closed, $Q$ open, and $Q \neq Z$;

$P^{+} \subset Y^{*}$ and $Q^{+} \subset Z^{*}$ are the nonnegative polar cones of $P$ and $Q$;

$S \subset Y \times Z$ is a given nonvoid set;

$V:=\{z \in Z \mid(y, z) \in S, y \in-P\}$ is convex, and for all $z^{*} \in Q^{+} \backslash\left\{0_{Z} \cdot\right\}$ the set

$D:=\left\{y \in Y \mid(y, z) \in S,\left\langle z^{*}, z\right\rangle<0\right\}$ is convex;

$S^{f}$ denotes the collection of all finite, nonempty subsets of $S$.

Note that $y \in-P$ and $y^{*} \in P^{+}$imply $\left\langle y^{*}, y\right\rangle \leq 0$, whereas $z \in-Q$ and $z^{*} \in Q^{+} \backslash\left\{0_{Z^{*}}\right\}$ imply $\left\langle z^{*}, z\right\rangle<0$. For simplicity we write $\{0\}$ instead of $\left\{0_{z *}\right\}$.

1 Fakultāt für Mathematik und Informatik, Universität Mannheim, D-6800 Mannheim 1, Germany 
Theorem 1. The system

$$
(y, z) \in S, \quad y \in-P, \quad z \in-Q
$$

has no solution if, and only if, there exists $z^{*} \in Q^{+} \backslash\{0\}$ with the property that

$$
\left\{\begin{array}{l}
\text { for all } \Omega \in S^{f} \text { there exists } y^{*} \in P^{+} \text {such that } \\
\left\langle z^{*}, z\right\rangle \geq 0 \text { for all }(y, z) \in \Omega \text { satisfying }\left\langle y^{*}, y\right\rangle \leq 0 .
\end{array}\right.
$$

Proof: a) Assume that (2) is satisfied with some $z^{*} \in Q^{+} \backslash\{0\}$. Then (1) cannot have a solution $(\bar{y}, \bar{z})$. Otherwise with $\Omega:=\{(\bar{y}, \bar{z})\}$ we would have for $y^{*} \in P^{+}$as given by (2) that $\left\langle y^{*}, \vec{y}\right\rangle \leq 0$ and thereby $\left\langle z^{*}, \vec{z}\right\rangle \geq 0$, whereas from $\bar{z} \in-Q$ and $z^{*} \in Q^{+} \backslash\{0\}$ follows $\left(z^{*}, \vec{z}\right)<0$, a contradiction.

b) Assume that (1) has no solution. Then the convex set $V$ introduced in the assumptions is disjoint from the open convex cone $-Q$. Hence by the weak separation theorem for convex sets there exists $z^{*} \in Q^{+} \backslash\{0\}$ such that $\left\langle z^{*}, z\right\rangle \geq 0$ for all $z \in V$ (if $V$ is empty, then choose $z^{*} \in Q^{+} \backslash\{0\}$ arbitrarily, which is possible since $\left.Q \neq Z\right)$. Then the system

$$
(y, z) \in S, \quad y \in-P, \quad\left\langle z^{*}, z\right\rangle<0
$$

has no solution. For the convex set $D$ introduced in the assumptions this means that $D \cap-P=1$. Let $\Omega$ be a finite, nonempty subset of $S$. Let $D_{\Omega}:=\{y \in Y \mid(y, z) \in \Omega$, $\left.\left\langle z^{*}, z\right\rangle<0\right\}$. If $D_{\Omega}=0$, then choose $y^{*}=0$. If $D_{\Omega} \neq 0$, then from the convexity of $D$ follows conv $D_{\Omega} \subset D$. Hence conv $D_{\Omega} \cap-P=\emptyset$. Since conv $D_{\Omega}$ is convex, compact and $P$ is convex, closed and $Y$ is locally convex, the strong separation theorem gives $y^{*} \in P^{+}$ such that $\left\langle y^{*}, y\right\rangle>0$ for all $y \in$ conv $D_{\Omega}$, hence for all $y \in D_{\Omega}$. So from $\left\langle y^{*}, y\right\rangle \leq 0$ and $(y, z) \in \Omega$ follows $y \notin D_{\Omega}$, i.e., $\left\langle z^{*}, z\right\rangle \geq 0$.

q.e.d.

We mention that in order to obtain in (2) the slightly stronger but more familiar "Lagrangian" statement $0 \leq\left\langle y^{*}, y\right\rangle+\left\langle z^{*}, z\right\rangle$ for all $(y, z) \in \Omega$, not only stronger convexity requirements are needed (e.g. $S$ convex), but also a regularity assumption - see [1].

Of particular interest is the case that

$$
S:=(f \times g)(X)+(P \times \mathrm{cl} Q),
$$

where $X$ is a convex set and $f: X \rightarrow Y, g: X \rightarrow Z$ are given mappings. With this specialization, since $P+P=P$ and cl $Q+Q=Q$, the inconsistency of (1) means the inconsistency of the system

$$
x \in X, \quad f(x) \in-P, \quad g(x) \in-Q .
$$

Statement (2) takes the following form :

$$
\begin{aligned}
& \text { For all } W \in X^{f} \text { there exists } y^{*} \in P^{+} \text {such that } \\
& \left\langle z^{*}, g(x)\right\rangle \geq 0 \quad \text { for all } x \in W \quad \text { satisfying }\left\langle y^{*}, f(x)\right\rangle \leq 0 .
\end{aligned}
$$

The convexity of $D$ resp. $V$ in this case is satisfied if for the multivalued mappings 
$\Psi(\cdot):=f\left(g^{-1}(\cdot)\right)+P$ resp. $\Psi(\cdot):=g\left(f^{-1}(\cdot)\right)+\mathrm{cl} Q$ one has that $\Psi(C)$ is convex for all convex subsets $C \subset Z$ resp. $C \subset Y$.

Motivated by [7] we give a vector-valued version of Theorem 1 .

Theorem 2. The system (1) has no solution if, and only if,

$$
\left\{\begin{array}{l}
\text { for all } \Omega \in S^{f} \text { there exists } y^{*} \in P^{+} \text {such that } \\
z \notin-Q \text { for all }(y, z) \in \Omega \text { satisfying }\left(y^{*}, y\right\rangle \leq 0
\end{array}\right.
$$

Proof: If (1) has no solution, then there exists $z^{*} \in Q^{+} \backslash\{0\}$ such that (2) is satisfied, and this implies (3) since otherwise $z \in-Q$ would imply $\left\langle z^{*}, z\right\rangle<0$. Conversely, let (3) hold. Then (1) has no solution $(\bar{y}, \bar{z})$. Otherwise we would set $\Omega:=\{(\bar{y}, \bar{z})\}$ and obtain from(3) an $y^{*} \in P^{+}$such that $\left\langle y^{*}, \vec{y}\right\rangle \leq 0$, hence $\bar{z} \notin-Q$. This contradicts $(\bar{y}, \bar{z})$ being a solution of (1). q.e.d.

3. Theorem 1 and Theorem 2 give rise to a duality theorem (compare [2] and [4]) in scalar and in vectorial form respectively. We first turn to the vectorial case, starting from Theorem 2. As before we let

$$
V:=\{b \in Z \mid(y, b) \in S, y \in-P\},
$$

and we let

$$
\begin{aligned}
& W:=\left\{b \in Z \mid \text { for all } \Omega \in S^{f} \text { there exists } y^{*} \in P^{+}\right. \text {such that } \\
& \left.\qquad z-b \notin-Q \text { for all }(y, z) \in \Omega \text { satisfying }\left\langle y^{*}, y\right\rangle \leq 0\right\} .
\end{aligned}
$$

$\bar{b} \in Z$ is called Pareto minimal in $V$ iff $\bar{b} \in V$ and $b-\bar{b} \notin-Q$ for all $b \in V$.

$\bar{b} \in Z$ is called Pareto maximal in $W$ iff $\bar{b} \in W$ and $b-\bar{b} \notin Q$ for all $b \in W$.

If $b_{1} \in V$ and $b_{2} \in W$, then $b_{1}-b_{2} \notin-Q$. Indeed: For $b_{1} \in V$ let $\left(y_{1}, b_{1}\right) \in S$ with $y_{1} \in-P$. Then for all $y^{*} \in P^{+}$we have $\left\langle y^{*}, y_{1}\right\rangle \leq 0$. In particular for the $y^{*} \in P^{+}$ resulting from $b_{2} \in W$ with $\Omega:=\left\{\left(y_{1}, b_{1}\right)\right\}$ we have $\left\langle y^{*}, y_{1}\right\rangle \leq 0$, implying $b_{1}-b_{2} \notin-Q$. From this it follows immediately :

If $\bar{b} \in V \cap W$, then $\bar{b}$ is Pareto minimal in $V$ and Pareto maximal in $W$.

Theorem s. $\bar{b} \in Z$ is Pareto minimal in $V$ if, and only if, $\bar{b} \in V \cap W$.

Proof: a) Assume that $\bar{b}$ is Pareto minimal in $V$. Then $\bar{b} \in V$, and the system

$$
(y, b) \in S, \quad y \in-P, \quad b-\bar{b} \in-Q
$$

has no solution. By Theorem 2 , where we have to replace $S$ by $S-(0, \bar{b})$, we obtain that $\bar{b} \in W$.

b) That $\bar{b} \in V \cap W$ implies $\bar{b}$ being Pareto minimal in $V$ has already been observed. q.e.d.

The scalar version is fully analogous. Again we let

$$
V:=\{b \in Z \mid(y, b) \in S, y \in-P\}
$$


and for $z^{*} \in Q^{+} \backslash\{0\}$ we let

$$
\begin{aligned}
& W\left(z^{*}\right):=\left\{b \in Z \mid \text { for all } \Omega \in S^{f} \text { there exists } y^{*} \in P^{+}\right. \text {such that } \\
& \left.\qquad\left\langle z^{*}, z-b\right\rangle \geq 0 \text { for all }(y, z) \in \Omega \text { satisfying }\left\langle y^{*}, y\right\rangle \leq 0\right\} .
\end{aligned}
$$

By a similar reasoning as above we obtain: If $b_{1} \in V$ and $b_{2} \in W\left(z^{*}\right)$, then $\left\langle z^{*}, b_{1}-b_{2}\right\rangle \geq 0$. From this it follows immediately :

$$
\text { If } \bar{b} \in V \cap W\left(z^{*}\right) \text {, then } \min _{b \in V}\left\langle z^{*}, b\right\rangle=\left\langle z^{*}, \bar{b}\right\rangle=\max _{b \in W\left(z^{*}\right)}\left\langle z^{*}, b\right\rangle \text {. }
$$

Theorem 4. $\bar{b} \in Z$ is Pareto minimal in $V$ if, and only if, there exists $z^{*} \in Q^{+} \backslash\{0\}$ such that $\bar{b} \in V \cap W\left(z^{*}\right)$.

Proof: The proof is analogous to that of Theorem 3. Note that from $\left\langle z^{*}, b-\bar{b}\right\rangle \geq 0$ and $z^{*} \in Q^{+} \backslash\{0\}$ follows $b-\bar{b} \notin-Q$. q.e.d.

For fixed $z^{*} \in Q^{+} \backslash\{0\}$ let us consider the quantities

There holds

$$
\alpha:=\min _{z \in V}\left\langle z^{*}, z\right\rangle \quad, \quad \beta^{*}:=\max _{z \in W\left(z^{*}\right)}\left\langle z^{*}, z\right\rangle .
$$

$$
\begin{aligned}
\beta^{*} & =\inf _{\Omega \in S^{+}} \sup _{y^{*} \in P^{+}}\left(\inf \left\{\left\langle z^{*}, z\right\rangle \mid(y, z) \in \Omega,\left\langle y^{*}, y\right\rangle \leq 0\right\}\right) \\
& \geq \sup _{y^{*} \in P^{+}} \inf _{\Omega \in S^{\prime}}\left(\inf \left\{\left(z^{*}, z\right) \mid(y, z) \in \Omega,\left\langle y^{*}, y\right\rangle \leq 0\right\}\right) \\
& =\sup _{y^{*} \in P^{+}}\left(\inf \left\{\left\langle z^{*}, z\right\rangle \mid(y, z) \in S,\left\langle y^{*}, y\right\rangle \leq 0\right\}\right) \\
& =: \beta .
\end{aligned}
$$

Hence in the situation of Theorem 4 one has $\alpha=\beta^{*} \geq \beta$. Under a suitable regularity assumption ( see [5], [6]) one has even $\alpha=\beta$. But without such a regularity assumption one may have a duality gap $\alpha>\beta$, and the value $\beta^{*}$ is designed so as to close eventually this gap.

\section{References}

[1] G. Heinecke, W. Oettli : A nonlinear theorem of the alternative without regularity assumption, J. Math. Anal. Appl. (to appear).

[2] J. Jahn : Mathematical Vector Optimization in Partially Ordered Linear Spaces. Frankfurt am Main, 1986.

[3] D. G. Luenberger : Quasi-convex programming, SIAM J. Appl. Math. 16 (1968), 1090-1095.

[4] W. Oettli : A duality theorem for the nonlinear vector-maximum problem, Colloquia Mathematica Societatis János Bolyai 12 (1974), 697-703.

[5] W. Oettli : Optimality conditions involving generalized convex mappings, in: Generalized Concavity in Optimization and Economics (ed. by S. Schaible and W. T. Ziemba), pp. 227-238. New York, 1981.

[6] I. Singer : Optimization by level set methods I : Duality formulae, in: Optimization (ed. by J.-B. Hiriart-Urruty, W. Oettli, J. Stoer), pp.13-43. New York, 1983.

[7] T. Weir, B. Mond, B. D. Craven : Weak minimization and duality, Numer. Funct. Anal. Optim. 9 (1987), 181-192. 